\title{
Shared susceptibility loci for breast, prostate and ovarian cancers
}

\begin{abstract}
Breast, prostate and ovarian cancers are sometimes grouped together because of the similar effects of estrogen or testosterone exposure on their progression, but little was previously known of their common genetic etiology. COGS found 18 regions containing variants associated with more than 1 of these cancers. For most of these, distinct loci within the same genomic region were identified as associated with different cancers, providing insights into shared mechanisms in the development of these hormone-related cancers.
\end{abstract}

\section{Orli Bahcall}

\section{Shared etiology for breast, ovarian and prostate cancers}

Breast and ovarian cancers share several lifestyle and environmental risk factors that are associated with endogenous and exogenous hormone exposure (Prog. Cin. Biol. Res. 396, 17-29, 1997). Although there are few established lifestyle risk factors for prostate cancer, it is also generally considered to be a hormone-related cancer. This shared etiology, in turn, raises the possibility that these and other cancers have common genetic pathways for susceptibility. It is clear that this is true for rare, high-penetrance cancer susceptibility allelesrare, deleterious variants of BRCA1 and BRCA2 are associated with greater risks of breast, ovarian and prostate cancers (J. Natl. Cancer Inst. 94, 1358-1365, 2002, Am. J. Hum. Genet. 72, 1117-1130, 2003 and Br. J. Cancer $105,1230-1234,2011)$. Whether this will also be true for common, low-penetrance susceptibility alleles is less clear. Cross-cancer familial risks are generally weak (PLoS Med. 1, e65, 2004), and most of the relative risks of ovarian and prostate cancers in multiple-case breast cancer families seem to be explained by BRCA1 and BRCA2 mutations. Although some less common variants in RAD51C were initially reported to be associated with both breast and ovarian cancers (Nat. Genet. 42, 410-414, 2010), subsequent studies have suggested that the risks are specific to ovarian cancer (Nat. Genet. 44, 475-476, 2012). Nevertheless, the identification of multiple different susceptibility loci for breast, ovarian and prostate cancers in the same genomic region, for example, at $8 q 24$, is consistent with the hypothesis of shared causal pathways, even if the causal mechanism underlying the association with each cancer remains to be elucidated. Although there is strong evidence that some common loci are associated with multiple cancers-for example, rs8170/rs2363956 at 19p13 is associated with ovarian cancer at genome-wide significance (Nat. Genet. 42, 880-884, 2010) and estrogen receptor (ER)-negative breast cancer at a less stringent significance level (Nat. Genet. 42, 885-892, 2010)—until now, no single SNP has been reported to be associated with the risk of more than one of these three cancers at an accepted level of genomewide statistical significance $\left(P<5 \times 10^{-8}\right)$.

In this summary, the related concepts of a shared genetic 'region' and a shared genetic 'locus' are considered distinct. A genetic region is defined as a contiguous part of the genome, which includes genomic DNA of anywhere from a few kilobases to many megabases in length. For the purposes of this discussion, we have arbitrarily chosen to designate a region as a block of genomic DNA spanning $2 \mathrm{Mb}$. A genetic locus is defined as a single, functional disease-associated allele. As such, each independent, disease-associated SNP is considered to be a locus. An example of this terminology is provided by the well-established region at $8 \mathrm{q} 24$ that includes multiple cancer susceptibility loci. 

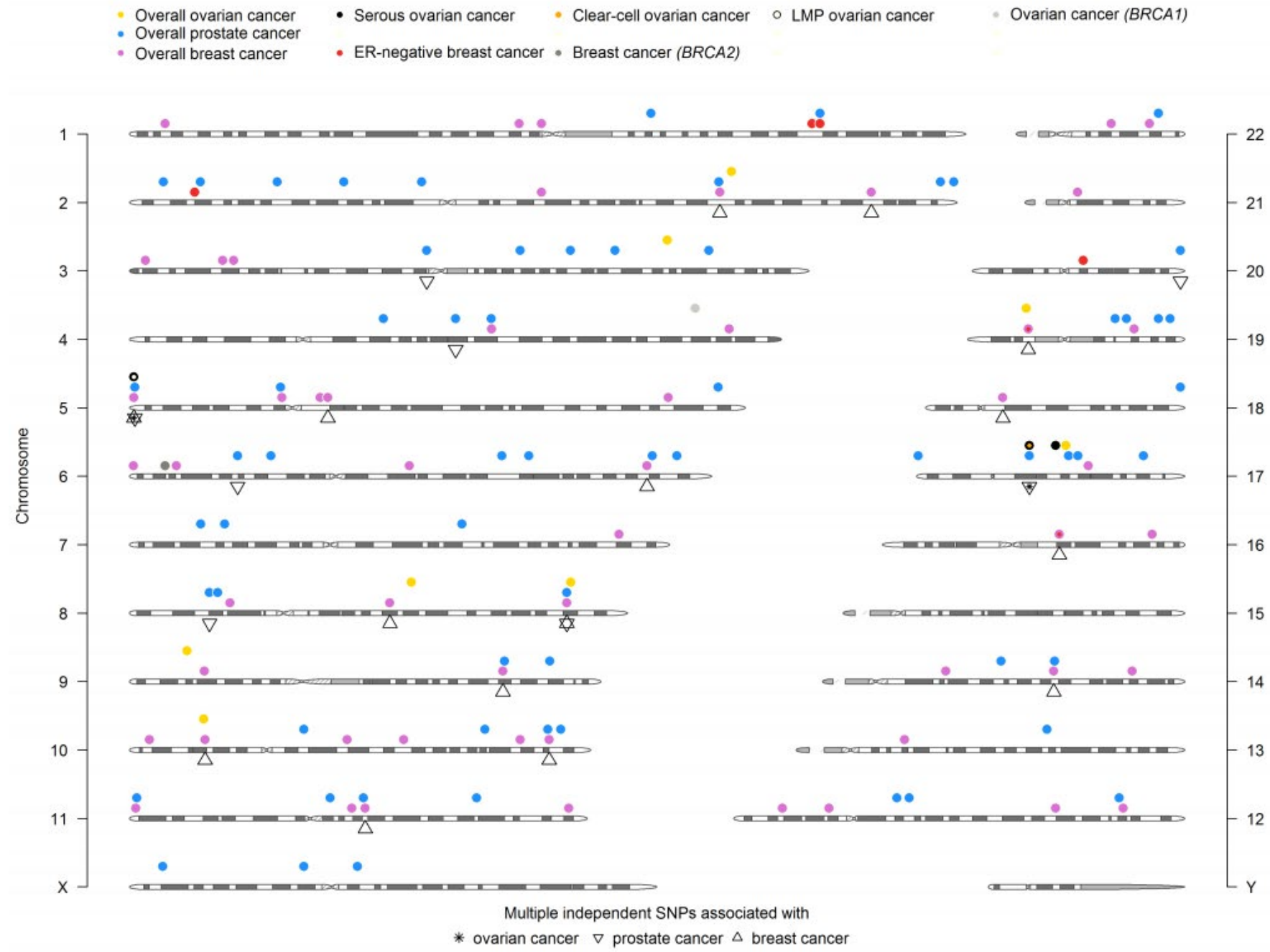

Figure 1 | Location of susceptibility loci identified for breast, prostate and ovarian cancers in the COGS studies. The chromosomal locations of the common breast, prostate and ovarian cancer susceptibility loci indentified so far are marked by colored circles above each chromosome. Regions with more than one independent locus for the same cancer (ignoring differences by subtype) are denoted by symbols below each chromosome; all regions contain two such loci, except $8 \mathrm{q} 24$, which contains six prostate cancer susceptibility loci, and 11 q13 and 16q12, which each contain three breast cancer susceptibility loci. Further details are provided in Table 1. The figure was created using the quantsmooth library in R statistical software.

\section{Multiple susceptibility regions for the same cancers}

On the basis of all previously reported and new loci identified by COGS, 15 regions (1q32.1, 2q31, 2q35, $5 q 11.2,6 q 25,8 q 21.11,8 q 24.21,9 q 31.2,10 p 12.31,10 q 26,11 q 13.1,14 q 24.1,16 q 12.1,18 q 11.2$ and 19p13.11) include more than 1 independent breast cancer susceptibility SNP within a 2-Mb region, such that each is associated with disease after adjustment for the others. There are three independently associated SNPs at both $5 q 11.2$ and 11q13.1. An additional two breast cancer risk loci lie within or close to known highpenetrance breast cancer susceptibility genes: rs11571833 is a polymorphic stop variant in BRCA2, and rs132390 lies $\sim 500 \mathrm{~kb}$ upstream of CHEK2, with its association persisting after adjustment for the CHEK2 c.1100delC mutation. Eight regions (3p12.1, 4q22, 5p15.11, 6p21, 8p21.2, 8q24.21, 17q12 and 20q13.33) harbor two or more independent loci associated with prostate cancer, and two regions (5p15.11 and 17q12) have two independent susceptibility loci for ovarian cancer. Many of the associations with breast cancer are specific to disease subtype defined by ER status, and, at 5p15.11,16q12 and 19p13.11, different SNPs are associated with risk of different subtypes (Nat. Genet. doi:10.1038/ng.2563, 27 March 2013 and Nat. Genet. 
doi:10.1038/ng.2561, 27 March 2013). Similarly, the ovarian cancer loci in the 5p15.11 and 17q12 regions are associated with different disease subtypes.

\section{Common susceptibility regions across cancers}

Eighteen regions spanning $2 \mathrm{Mb}$ included loci associated with more than 1 cancer: 12 (1q32.1, 2p24, 2q31, 4q24, 5p12, 6q25, 9q31, 10q26, 11p15.5, 11q13.3, 12q24 and 14q24) contained breast and prostate cancer susceptibility loci, 2 contained breast and ovarian cancer susceptibility loci (10p12.31 and 19p13.11); 2 contained prostate and ovarian cancer susceptibility loci (17q12 and 17q21.32); and 2 contained risk loci for all 3 cancers (5p15.11 and 8q24.21). In only two instances was the same SNP associated with risk of more than one cancer: rs4245739 (1q32.1) is associated with both breast and prostate cancers, and rs8170 (19p13) is associated with both ER-negative breast cancer and ovarian cancer.

Table 1 Breast, ovarian and prostate cancer susceptibility loci confirmed at genome-wide significance $(P<5 \times$ $\left.10^{-8}\right)$.

\section{View table (PDF)}

\section{Insights into mechanism}

The studies in the COGS collection have confirmed that there are genomic regions of susceptibility shared between breast, prostate and ovarian cancers. In most cases, the COGS studies have identified distinct loci within the same genomic region that are associated with different cancer types. One explanation for this finding could be that different variants affect regulatory elements that act on the same gene located nearby, for example, tissue-specific enhancers interacting with the promoter of the same gene. Alternatively, related genes may be arranged in tandem so that multiple loci affect multiple genes with related functions. Although only a handful of loci have been found that are clearly associated with risk of different cancers, it is possible that uncorrelated loci in the same region that affect different cancers might be acting through the same causal variant. Future cross-cancer analyses of the COGS data should help clarify this, but further fine mapping involving genotyping of a large number of SNPs across each region in each cancer will be needed, such as will be pursued by studies with the OncoChip (see Future plans and the OncoChip). Such fine mapping will be further enhanced by systematic functional studies to characterize the molecular mechanisms underlying cancer risk. 